Revista do Departamento de Geografia
Universidade de São Paulo
www.revistas.usp.br/rdg

\title{
Avaliação Espacial da Dengue na Área Urbana de Itu - São Paulo
}

\section{Assessment of the Spatial of Dengue in Urban Area of Itu - São Paulo}

\author{
Helio Henrique Ferreira \\ Universidade Estadual Paulista Júlio de Mesquita Filho - Sorocaba \\ heliohenrique@yahoo.com \\ Elfany Reis do Nascimento Lopes \\ Universidade Estadual Paulista Júlio de Mesquita Filho - Sorocaba \\ elfanyl@ hotmail.com \\ José Carlos de Souza \\ Universidade Estadual Paulista Júlio de Mesquita Filho - Sorocaba \\ jcsouza1974@gmail.com \\ Jocy Ana Paixão de Sousa \\ Universidade Estadual Paulista Júlio de Mesquita Filho - Sorocaba \\ jocypet@gmail.com \\ Roberto Wagner Lourenço \\ Universidade Estadual Paulista Júlio de Mesquita Filho - Sorocaba \\ robertow@sorocaba.unesp.br
}

Recebido (Received): 18/03/2017 DOI: $10.11606 /$ rdg.v33i0.128515

Aceito (Accepted): 07/05/2017

Resumo: O Brasil tem vivenciado um aumento progressivo e alarmante de casos de dengue nos últimos anos. Estudar a distribuição espacial e temporal de casos de dengue e de infestação larvária em áreas urbanizadas é relevante para reconhecer padrões de concentração de casos e de localização de maior incidência dos vetores, direcionando ações de vigilância epidemiológica. Os casos confirmados de dengue autóctone e importados e o índice de infestação predial no período de 2005 a 2014 na cidade de Itu (São Paulo) foram investigados. O trabalho foi realizado utilizando técnicas de geoprocessamento para cálculo da incidência da doença por bairro, obtenção do mapa de risco relativo e a determinação da autocorrelação espacial entre os casos de dengue através dos Índices Local e Global de Moran. O ano de 2013 apresentou o maior registro e notificação de casos autóctones e importados de dengue. As maiores taxas de casos e de incidência média encontram-se na parte central e noroeste, sendo as áreas periféricas e limítrofes destas regiões com ocorrências significativas. Nos bairros a dengue variou entre 0 e 119 casos, sendo a maior concentração na região central. A incidência média de dengue encontra-se elevadas na parte central e noroeste, com valor máximo de 11.9 a cada 100 mil habitantes. Observou-se uma autocorrelação espacial entre áreas centrais e periféricas, enquanto o risco relativo da doença possui maior predomínio na parte central, mas não permite afirmar a ausência do risco de ocorrências nas demais áreas.

Palavras-chave: Saúde Pública; Aedes aegypti; Controle de Vetores; Epidemiologia; Geoprocessamento
Abstract: Brazil has experienced a progressive and alarming increase of dengue cases in recent years. Studying the spatial and temporal distribution of dengue cases and larvae infestation in urbanized areas is relevant to recognition patterns of cases and location of the higher incidence of vectors, directing epidemiological surveillance actions. The confirmed cases of autochthonous and imported dengue and the infestation rate in the period from 2005 to 2014 in the city of Itu (São Paulo) were investigated. The work was performed using geoprocessing tools to calculate the incidence by neighborhood, obtaining the relative risk map and the determination of the spatial autocorrelation between cases of dengue through the Local and Global Moran Indices. The year 2013 presented the highest notification and registry of autochthonous and imported cases of dengue, being the highest rates were founded at the central and northwest part with significant occurrences. In the districts dengue ranged from 0 to 119 cases, with the highest concentration in the central region. The average incidence of dengue fever is high in the central and northwestern part, with a maximum value of 11.9 per 100.000 inhabitants. In addition, there was a spatial autocorrelation between central and peripheral areas, while the relative risk of the disease has a higher prevalence in the central part, but does not induce the absence of risk occurrences in other areas.

Keywords: Public Health; Aegypti Aedes; Vector Control; Epidemiology; Geoprocessing 


\section{INTRODUÇÃO}

A dengue é uma da doença tropical e sub-tropical, cuja infecção viral sistêmica tem como agente etiológico o vírus DENV, que é transmitido pela picada do mosquito infectado do gênero Aedes, sendo o Aedes aegyptio principal vetor da transmissão da doença. Conhecida internacionalmente pelo Código Internacional de Doença (CID10) como A90, o conhecimento da distribuição geográfica dos casos de dengue nos espaços urbanos é essencial para o entendimento da mortalidade causadas pela patologia e auxiliar as políticas de gestão e avaliação do impacto da doença sobre a população (GUZMAN et al., 2010; TELLE, 2015).

Estima-se que entre 70 a 500 milhões de pessoas são infectadas pelo vírus da dengue a cada ano, em mais de cem países, sendo que a maioria dos casos ocorre em áreas urbanas (SIMMONS et al., 2012; BHATT et al., 2013; GONÇALVES et al., 2015; TELLE, 2015). As taxas de incidência são maiores na América Latina e Ásia, enquanto a África possui as informações mais escassas sobre a patologia. Na Índia, as maiores prevalências foram detectadas em áreas urbanizadas e com predomínio de infecção em homens entre 26 e 59 anos (BHATT et al., 2013; TELLE, 2015).

Já no Brasil, estudos realizados no estado do Rio de Janeiro têm evidenciado um aumento significativo dos focos de mosquito A. aegypti em área urbanas (HONORIO, 2010). No estado de São Paulo, a disseminação é evidente em áreas de ocupação irregular e com precária infraestrutura de saneamento básico (MONDINI, 2009; BARBOSA; LOURENÇO, 2010; ARAÚJO et al., 2015).

A dengue é um problema de saúde pública no Brasil e suas estatísticas apontam a mudança no perfil epidemiológico, com aumento no número de casos graves e acometimento de jovens. Os casos de dengue no país cresceram de 40.279 em 1990 para 589.100 casos em 2014. No entanto, no ano de 2013 houve um surto epidêmico da doença, com um total de 1.452 .489 casos, sendo as regiões Sudeste e Centro-Oeste líderes nas estatísticas por regiões brasileiras (BRASIL, 2015).

O aumento progressivo dos casos de dengue no país é resultado de múltiplos fatores socioambientais que incluem desde a ausência de educação ambiental, baixa vigilância epidemiológica, destinação incorreta de resíduos sólidos até o saneamento básico precário, colaborando para o avanço da patologia. Esses fatores estão combinados com o crescimento populacional e urbano aliado à desinformação dos moradores quanto à necessidade de eliminar os focos de proliferação, potencializando a ocorrência de dengue nos espaços urbanos (GLUBER, 1997; SILVA; MIRANDA, 2003; BARBOSA; SILVA, 2015; PELISSARI, 2016).

O uso de técnicas que avaliam a sazonalidade e a espacialidade no entendimento e vigilância da dengue, tem se tornado tendência e são abordagens potenciais para compreender o quadro da evolução da doença e sua distribuição geográfica. Esta abordagem associada aos Sistemas de Informações Geográficas (SIG) e ao geoprocessamento tem permitido o monitoramento das epidemias de forma especializada, contribuindo na tomada de decisões no âmbito das políticas públicas (LIMA et al., 2003; BARBOSA; SILVA, 2015; WHE et al., 2015; LIMA, 2016).

Este estudo contribui para o conhecimento do padrão de distribuição temporal e espacial da doença, considerando a importância do controle da dengue no cenário nacional e as séries de casos na região sudeste do Brasil. Assim, objetivou-se analisar a distribuição espacial e temporal dos casos de dengue autóctones e importados no período de 2005 a 2014, no espaço urbano da cidade de Itu, São Paulo.

\section{MATERIAIS E MÉTODOS}

O estudo foi desenvolvido no município de Itu, localizado na região centro-oeste do Estado São Paulo. A área territorial corresponde a $640,719 \mathrm{~km}^{2}$, densidade demográfica de 241,01 hab. $/ \mathrm{km}^{2}$ e 154 bairros distribuídos entre a zona urbana e rural, sendo a população estimada em 2016 de 167.095 habitantes. O clima do município é caracterizado por ser Subtropical Úmido (Cwa), segundo a classificação climática de Köppen, com chuvas no verão e seca no inverno. Temperatura média superior a $22^{\circ} \mathrm{C}$ e com totais anuais de precipitação em torno de 1300 mm (PREFEITURA DE ITU, 2015; IBGE, 2016; SEADE, 2015). 
O estudo realizado é do tipo exploratório da distribuição espacial dos casos de dengue autóctones e importados confirmados através de exame laboratorial, cujas estatísticas estão disponíveis no Centro de Controle de Zoonoses do município, agrupados por ano de ocorrência e bairro. O setor segue normas do Plano Nacional de Controle da Dengue (PNCD) e promove a visita dos imóveis do município para avaliação dos índices de infestação, além do atendimento de casos suspeitos, buscando o controle da doença (BRASIL, 2002). Foram analisados 93 bairros com notificação dos casos de dengue para o período de dez anos (2005 a 2014).

Foi calculada a Taxa de Incidência Média dos casos de dengue a cada 100.000 habitantes para o município, através da razão entre o número total de casos de dengue por ano (2005 a 2014) e a taxa populacional do município segundo dados da Fundação Sistema Estadual de Análise de Dados de São Paulo (SEADE), da seguinte maneira:

$$
T I M=(\mathrm{Nb} / \mathrm{Pb}) \times 100.000 \mathrm{hab} .
$$

Sendo:

$\mathrm{T}_{\mathrm{IM}}$ - taxa média da incidência de dengue por município;

$\mathrm{Nb}$ - número de casos de dengue por ano (2005 a 2014);

$\mathrm{Pb}$ - população total do município por ano (2005 a 2014).

Foi calculada a Taxa de Incidência Média dos casos de dengue a cada 1.000 habitantes por bairro, através da razão entre o número de casos por ano (2005 a 2014) em cada bairro e a população residente no bairro, segundo dados obtidos no censo demográfico do Instituto Brasileiro de Geografia e Estatística (IBGE, 2010), como a seguir:

$$
T I M B=(\mathrm{Nb} / \mathrm{Pb}) \times 1.000 \mathrm{hab} .
$$

\section{Sendo:}

TIMB - taxa média da incidência de dengue por bairro;

$\mathrm{Nb}$ - número de casos de dengue por ano em cadabairro;

$\mathrm{Pb}$ - população total do bairro a cada ano (2005 a 2014).

Para verificar se há dependência espacial global entre os bairros com relação a taxa de incidência no período, foi utilizado o Índice de Moran Global (IMG). O IMG é uma medida cuja hipótese nula é de independência espacial, sendo que os valores positivos $>0$ e 1 indicam correlação direta, ou seja, valor do atributo de um objeto tende a ser semelhante aos valores dos seus vizinhos e os valores negativos entre $<0 \mathrm{e}$ -1 indica uma correlação inversa ${ }^{23}$. Sua equação é dada por:

$$
I=\frac{n}{\sum_{i=1} n \sum_{i=1}^{n} w_{i j}} \frac{\sum_{i=1} n \sum_{i=1}^{n} w_{i j}\left(y_{i}-y\right)\left(y_{i}-y\right)}{\sum_{i=1} n\left(y_{i}-y\right)^{2}} \text { para } i \neq j
$$

Sendo:

I - Índice de Moran Global para casos de dengue;

n - número de áreas (bairros);

$\mathrm{y}_{\mathrm{i}}-$ valor do atributo considerado na área $i$;

$\mathrm{y}_{\mathrm{j}}$ - valor do atributo considerado na área $j$;

y - valor médio do atributo na região de estudo;

$\mathrm{w}_{\mathrm{ij}}$ - pesos atribuídos na determinação da matriz de proximidade conforme a conexão entre as áreas $i$ e $j$.

Já a dependência espacial local dos casos de dengue e da taxa de incidência foi verificada através do Índice de Moran Local (LISA). Este procedimento é similar ao IMG, mas agrupa os bairros a partir da similaridade entre ambos (clusters), bairros com prevalências anômalas (outliers) ou com ausência de semelhança (SILVA et al., 2014). O índice é dado pela seguinte equação: 


$$
\mathrm{I}_{i}=\frac{\left(y_{i}-y\right) \sum_{i} i=1\left(y_{j}-y\right)}{\frac{\sum_{i=1} n\left(y_{\mathrm{I}}-y\right)^{2}}{n}}
$$

Sendo:

$\mathrm{I}_{\mathrm{i}}$ - índice de Moran Local;

n - número de áreas (bairros);

$\mathrm{y}_{\mathrm{i}}-$ valor do atributo considerado na área $i$;

$\mathrm{y}_{\mathrm{j}}$ - valor do atributo considerado na área $j$;

y - valor médio do atributo na região de estudo;

$\mathrm{W}_{\mathrm{ij}}$ - pesos atribuídos na determinação da matriz de proximidade conforme a conexão entre as áreas $i$ e $j$.

Os casos de dengue e os valores de incidência de dengue por bairro foram lançados em uma base cartográfica digital georreferenciada em um sistema plano cartesiano, para tratamento por meio de técnicas de geoprocessamento. Foi utilizado o software ArcGis 10.3 (ESRI, 2014) na construção dageocodificação das informações, ligando dadospoligonais vetoriais dos bairros, com as tabelas contendo as informações do número de casos por bairro/ano e dos índices de autocorrelação espacial. As informações foram conectadas tendo como referência de localização espacial o centro geométrico de cada um dos bairros.

Aplicou-se a análise da distribuição espacial por Kernel para análise da densidade de casos de dengue por bairro no período do estudo. A distribuição espacial por Kernel é entendida como uma técnica que calcula a densidade de recursos por unidade de área, onde seja $S$ uma localização geral em $R$ e $s_{1}, s_{2}, \ldots, s_{n}$ as localizações de $n$ eventos observados, então a intensidade $\lambda(s)$ (BRASIL, 2005; BARBOSA; LOURENÇO, 2010). O estimador é dado pela seguinte equação:

$$
\lambda_{\tau}^{n}(s)=\frac{1}{\delta_{\tau}(s)} \sum_{i=1} n \frac{1}{\tau^{2}} k\left[\frac{\left(s-s_{i}\right.}{\tau}\right] y_{i}
$$

Sendo:

$\lambda \tau(\mathrm{s})$ - valor estimado por área;

$\delta_{\tau}(\mathrm{s})$ - valor entre 0 e 1 que representa uma correção de borda;

$\tau$ - fator de suavização;

$\mathrm{s}$ - centro de cada espaço da grade regular;

$\mathrm{s}_{\mathrm{i}}$ - local de ocorrência do evento;

$\mathrm{k}$ - função de densidade de probabilidade.

$\mathrm{y}_{\mathrm{i}}$ - valor do evento no ponto, ou seja, o indicador da positividade de recipientes.

A densidade larvária do mosquito foi estuda espacialmente a partir do Índice de Infestação Predial (IIP), obtido do Levantamento de Índice Rápido para Aedes aegypti (LIRAa) do Sistema de Vigilância do município (BRASIL, 2005). O IIP corresponde à proporção de imóveis com larvas em relação ao total de imóveis inspecionados, classificado em satisfatório $(<1 \%)$, situação de alerta $(1 \%$ a $3,9 \%)$ e risco de surto $(>3,9 \%)$. Para se obter o IIP utiliza-se a seguinte equação:

\section{$I I P=($ Imóveis rurais $/$ Imóveis inspecionados $) \times 100$}

A elaboração do mapa de risco relativo de dengue por bairro para o período ente 2005-2014 foi obtido por meio da combinação dos casos de dengue por bairro/ano, do TIMB e IIP, conforme o calculo a seguir:

$$
R R d b=(T I M B \times 0.20)+(I I P \times 0.50)+(C D \times 0.30) / 3
$$

Sendo:

RRdb - Risco relativo de dengue por bairro;

TIMB - Taxa de incidência de casos de dengue por bairro;

IIP - Índice de infestação predial por bairro;

$\mathrm{CD}$ - Casos de dengue por bairro. 
Os valores do risco relativo foram obtidos em uma coluna adicional a tabela vinculada ao vetor do arquivo digital dos bairros, através da ferramenta de cálculo presentes no software ArcGis 10.3 e sua representação espacial através de um mapa temático de risco relativo para o município.

\section{RESULTADOS E DISCUSSÃO}

No período de dez anos foram registrados 1379 casos de dengue em Itú, sendo 1214 autóctones (88\%) e 165 importados (12\%). No ano de 2013 ocorreu a maior incidência, coincidente com o pico de casos ocorridos no país. Neste mesmo ano, todo o território brasileiro foi acometido por uma epidemia da doença, registrando 1.450.000 casos (BRASIL, 2015). O Estado de São de Paulo apresentou neste ranking, 208.914 casos e para 2015 esses números equivaleram a 975.505 casos, 63\% do total do país (PEREIRA et al., 2013; BRASIL, 2015).

A série do total de casos autóctones e importados confirmados evidenciou que os anos de 2011 e 2013 egistraram as maiores ocorrências, representando cerca de $63 \%$ do total de casos.

Foi verificado ainda, um crescimento progressivo da doença a partir de 2006, com picos elevados de 2006 para 2007, de 2008 para 2009 e de forma significativa de 2012 para 2013. Em outras cidades do Estado de São Paulo essas notificações também foram progressivas entre 2006, 2007 e 2014, o que reforça o problema de saúde pública no Estado e, consequentemente, no Brasil. (PEDROSO; MENDES, 2011; JOHANSEN et al., 2014).

A taxa de incidência média, a cada 100 mil habitantes, referente aos casos confirmados na área de estudo apresentam um crescimento progressivo no período analisado, evidenciando o agravamento da doença nos dias atuais (Figura 1).

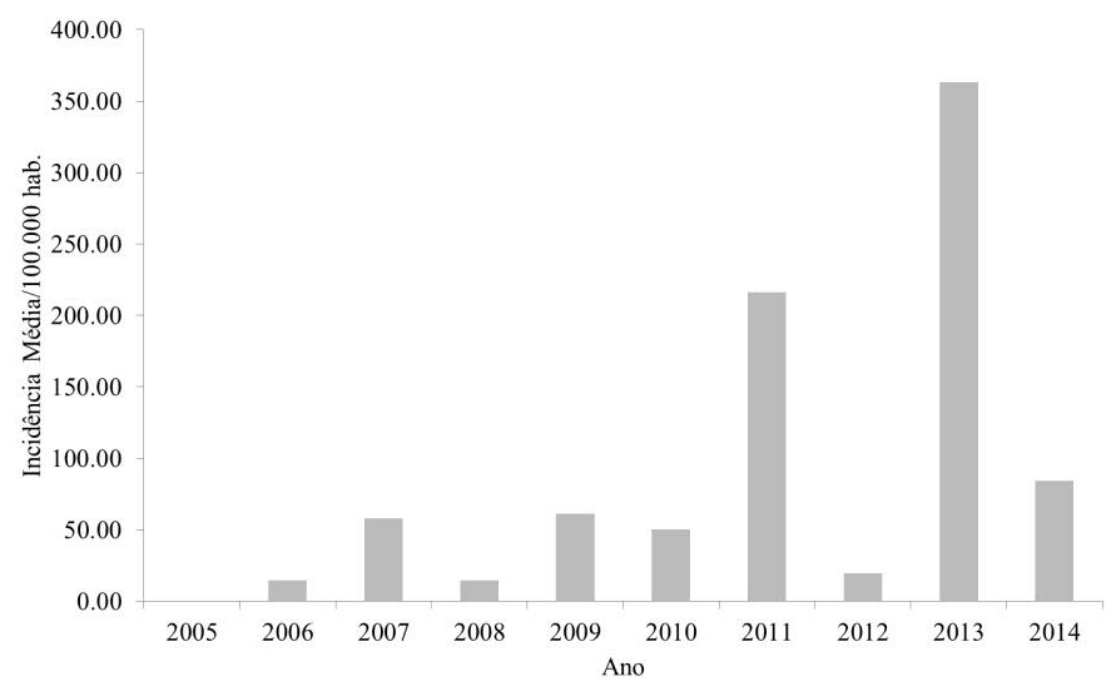

Figura 1: Taxa de incidência média da dengue a cada 100 mil habitantes, na cidade de Itú, São Paulo, entre 2005 e 2014.

Os resultados de 2013 superam a taxa de incidência média encontrada para a cidade de Campinas para o mesmo ano, correspondente a 300 casos/100 mil habitantes (JOHANSEN et al., 2014).

De acordo com as projeções dos órgãos de saúde do país, essa tendência de crescimento já era esperada, principalmente no Estado de São Paulo, por ser na região sudeste do Brasil, o Estado com maior destaque em número de ocorrências. Esse crescimento se deve principalmente ao baixo investimento em saúde pública, combate aos focos e a ausência de políticas públicas voltadas ao controle dos vetores do mosquito, aliados a falta de planejamento urbano e de saneamento básico, que associados, torna a doença um problema multifatorial no Brasil (BRASIL, 2014; 2015; 2016). 
Por outro lado, os casos de autoctonia registrados para os bairros estão relacionados à precariedade da infraestrutura urbana com ausência de saneamento e coleta adequada de lixo identificada na área de estudo.Esses mesmos apontamentos são verificados em outros estudos na região sudeste, onde, no Estado do Espírito Santo a maior incidência de dengue esteve relacionada com o destino inadequado do lixo, e em Minas Gerais, a epidemia foi associada à falta de saneamento básico e de infraestrutura urbana (HONORATO et al., 2014; HORTA et al., 2014).

As ações voltadas para o controle do foco do vetor também são eficientes ao combate à doença, dentre elas, a mobilização da sociedade e intervenções do governo em locais em que há grande dificuldade de acesso, a destinação adequada do lixo e o monitoramento do vetor, mas é imprescindível o engajamento da população para que as medidas sejam efetivas (REIS et al., 2013; HONORATO et al., 2014).

Na distribuição espacial dos casos de dengue por bairros, observada na Figura 3, onde, a concentração de casos ocorre principalmente em bairros centrais e em áreas periféricas das regiões oeste e sudoeste da cidade, variando entre 61 e 119 casos. As menores concentrações foram observadas na região leste e norte, com bairros apresentando um único caso, podendo ser observadas na Figura 2.

A incidência média a cada 1.000 habitantes por bairro evidenciou um padrão de distribuição espacial com maior concentração na região central e noroeste, enquanto uma baixa incidência foi verificada para bairros periféricos do município, conforme aponta a Figura 3.

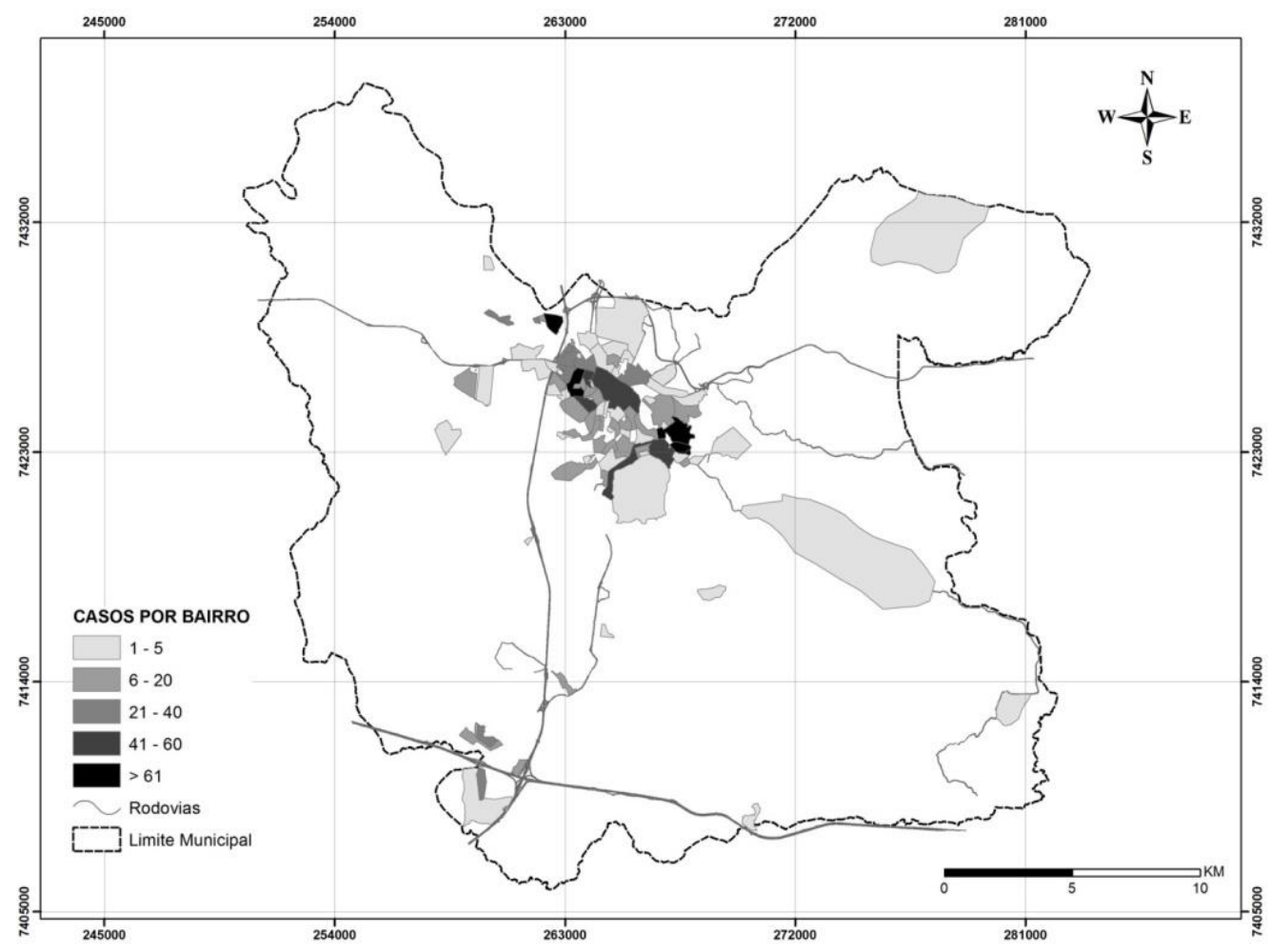

Figura 2: Casos de dengue por bairro na cidade de Itu, São Paulo, entre 2005 e 2014.

As taxas elevadas nessas regiões podem estar relacionadas ao fator da área central ser altamente urbanizada e possuir alto fluxo de circulação de pessoas devido a sua localização estar na divisa com os municípios de Salto, Boituva e Sorocaba. Essa condição também é potencializada pelo fato do município de Itú compor a região metropolitana do Estado de São Paulo.

A estrutura sanitária também pode representar um indicador direto para os casos de dengue. Embora a distribuição de água canalizada atenda a $100 \%$ da população urbana os distritos da zona rural possuem abastecimento por poços rasos e o tratamento de esgoto e resíduos sólidos é deficitário. As áreas ao norte e noroeste que incluem os bairros Vila Martins, Cidade Nova, Parque Novo Mundo, Jardim União e Vila Vivenda e ao sul no Portal do Éden, Village Castelo e City Castelo 2, apresentam despejo de esgoto sem tratamento diretamente nos corpos hídricos (SSE/CSAN, 2010). 
A coleta e disposição final dos resíduos sólidos atendem a 98\% da população, mas o sistema de varrição é operado apenas em 50\% da área urbana do município, podendo potencializar o armazenamento impróprio de água em resíduos e favorecer o desenvolvimento do vetor (SSE/CSAN, 2010). Assim, como a ausência de casos, a região sudeste do município também apresenta ausência de notificação de incidência média da doença (Figura 3).

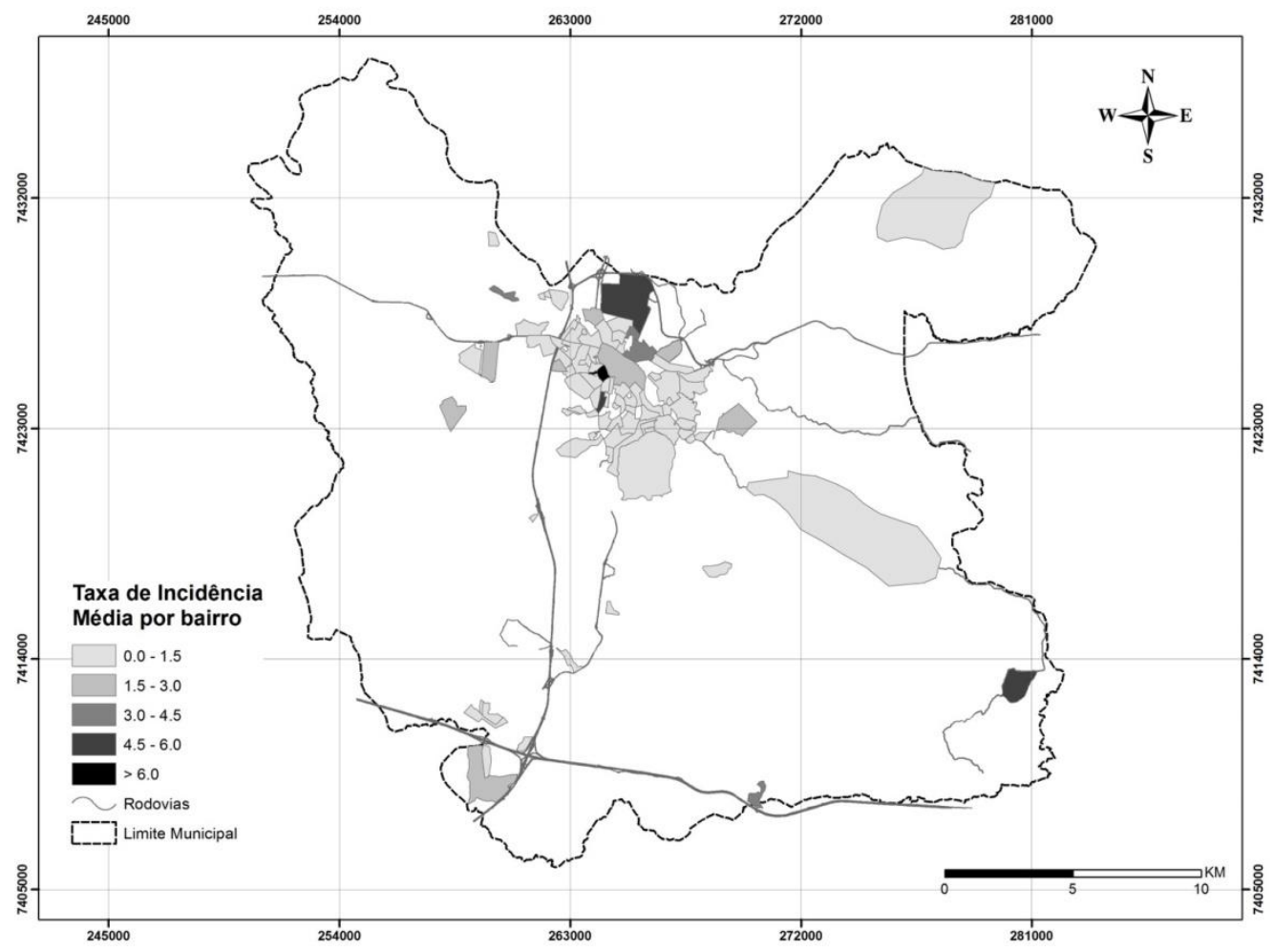

Figura 3: Taxa de incidência média 1000hab/bairro na cidade de Itu, São Paulo, entre 2005 e 2014.

De uma forma geral, ficou evidente que a incidência média e os casos de dengue por bairros apresentam maiores taxas na região central e noroeste, ou seja, ao longo dos anos, essas regiões apresentaram os maiores quantitativos de casos e incidência, enquanto que a distância dos agloremados centrais é proporcional à redução dessas taxas. A mesma observação foi realizada em outros estados do Brasil, onde em um período de 10 anos verificou-se que $97,34 \%$ casos de dengue ocorreram em pessoas que residiam em áreas urbanas (NASCIMENTO et al., 2015).

O reconhecimento do padrão de distribuição espacial da incidência de dengue no perímetro urbano, medido pela magnitude da dependência espacial do Índice Global de Moran (GISA) para os dez anos estudados, apontaram ausência de correlação entre os bairros, tendo os índices variando entre -0.0218 a 0.0450 .

A similaridade das incidências (clusters) verificada pelo Índice de Moran Local (LISA) indicou uma autocorrelação espacial com a formação de clusters para bairros da região central, com incidência média de 4.79. Já dois bairros localizados na região central e outro na região periférica sudoeste, obtiveram incidência média correspondente a 8.55 e 0.07 , respectivamente, sendo considerados outliers. Em contrapartida, a região periférica da área de estudo apresentou ausência de significância e autocorrelação espacial, como já observados nas demais taxas apresentadas.

Em trabalho similar no Estado do Mato Grosso, o LISA indicou os municípios com maior similaridade para a patologia, indicando assim as áreas com transmissão da doença mais concentrada no tempo. (FERNANDES et al., 2014). Neste estudo, o LISA indicou que os bairros centrais são mais similares ao longo do tempo em torno da incidência da doença no município, revelando ser uma área crítica com potencial para intervenção dos órgãos de saúde. 
A Figura 4 apresenta a densidade temporal e espacial de casos de dengue. É possível observar um padrão de alta incidência de distribuição da dengue também na região central da área de estudo e taxas elevadas a noroeste.

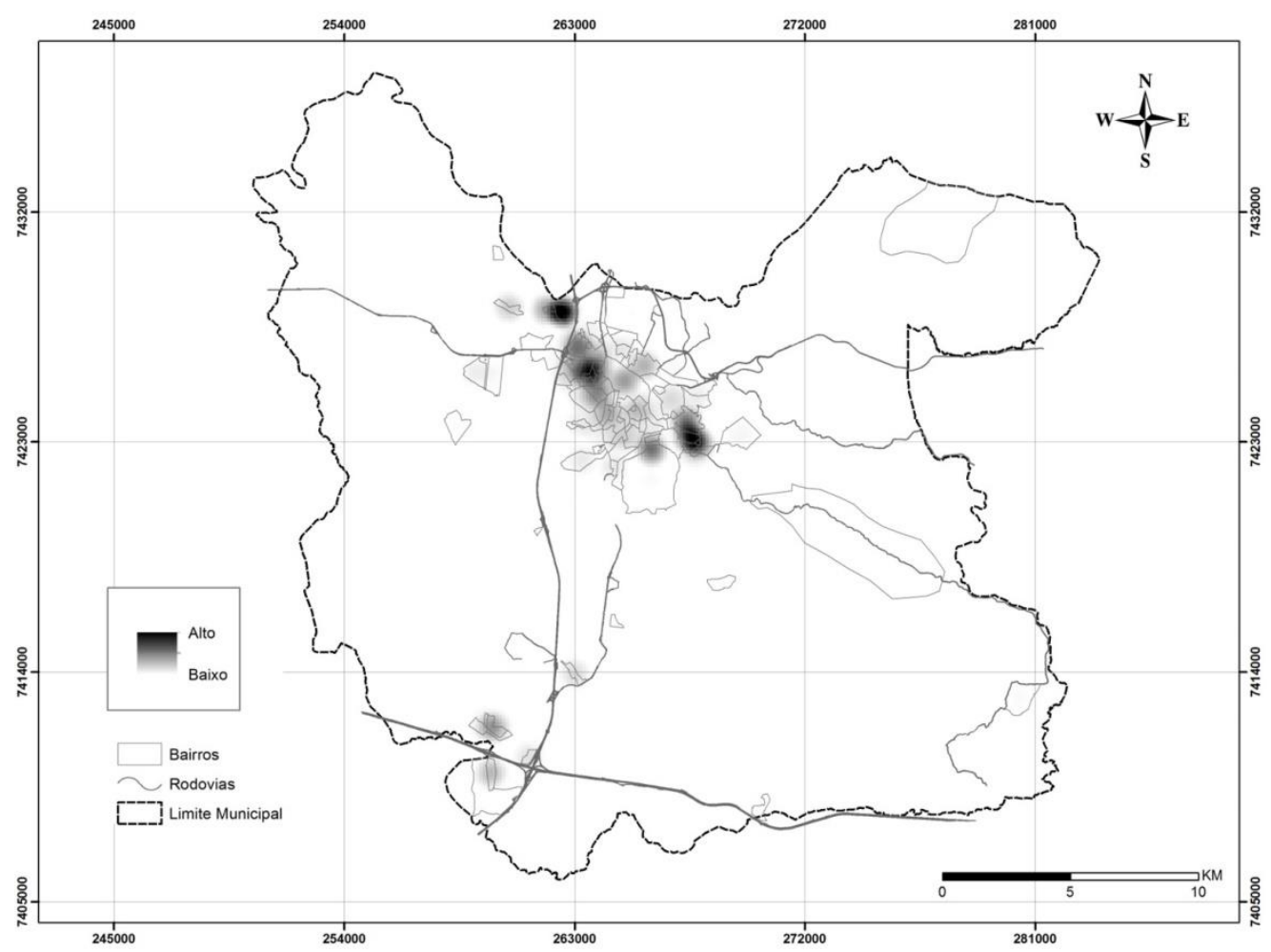

Figura 4: Densidade espacial e temporal dos casos de dengue entre 2005 e 2014 em Itu, São Paulo, entre 2005 e 2014.

Observou-se a formação de três aglomerados principais, denotando os bairros com maiores necessidades de intervenções e revisitas para averiguações da disseminação do mosquito e de seus focos. Considerando que esses aglomerados se distribuem em três pontos aproximados, os mesmos contribuem para irradiar a doença pelo seu entorno, já que, a fiscalização em um bairro e o descuido em outro próximo, não auxilia o combate da doença.

Embora as áreas com tendência a baixa incidência da dengue, encontrem-se associadas à distância da faixa central, afirma-se que independentemente da localização do bairro, a dengue pode se expandir por regiões diferentes daquelas de origem, além de que há alta probabilidade de uma pessoa que mora em um local sem infestação de se contaminar em outras áreas (SOUZA; BARATA, 2012; NASCIMENTO et al., 2015).

A média do Índice de Infestação Predial (IIP) permitiu classificar todos os bairros como satisfatórios. Contudo, é preciso reforçar a fiscalização nos bairros de modo a controlar os focos e a incidência da doença. O risco relativo a dengue por bairro apresentado na Figura 5, mostra que as áreas centrais possuem o maior risco de indivíduos contraírem a dengue.

O maior risco nessa área está relacionado ao predomínio de casos de dengue confirmados ao longo dos anos e de áreas infestadas com larvas, predispondo o desenvolvimento do vetor.

É preciso ressaltar que regiões que se distribuem fora da zona central possuem um baixo risco relativo para a doença, contudo, a disseminação é bastante complexa e mesmo estas regiões podem ser consideradas vulneráveis, uma vez que, apresentam em seu entorno bairros com incidência de ocorrência da doença e notificação do IIP. As mesmas observações foram verificadas nos estudos de Hassan et al. (2012) e de Pedroso e Moura (2016), quando indicaram um maior grau de risco de dengue em áreas centrais e no seu entorno. 


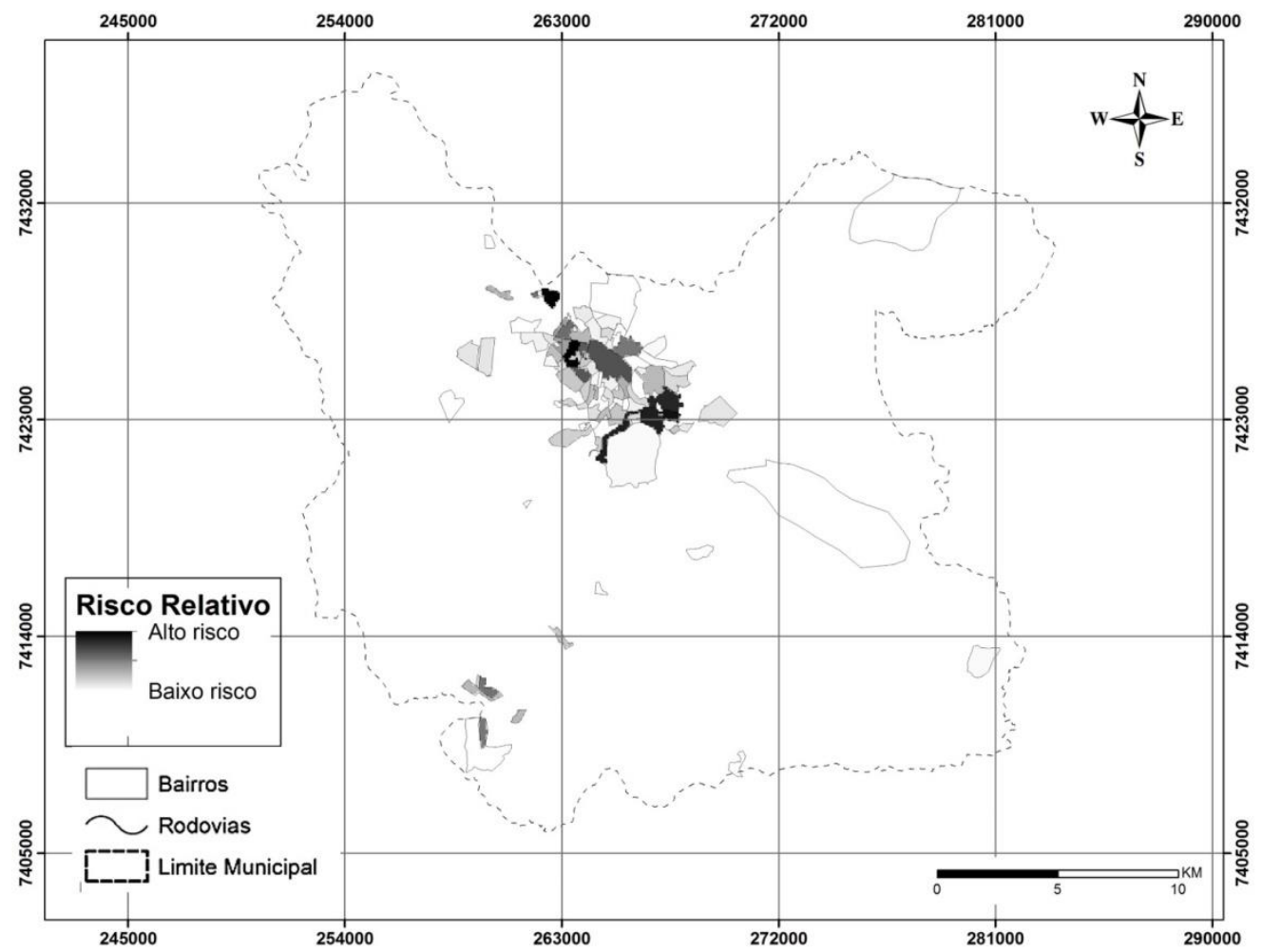

Figura 5: Risco relativo da ocorrência de dengue na cidade de Itu, São Paulo, entre 2005 e 2014.

\section{CONCLUSÃO}

A faixa central do perímetro urbano do município de Itu concentra a maior incidência de dengue. O aglomerado de casos justifica a concentração de infestação da doença sobre essas áreas, mas o risco relativo permite afirmar que mesmo os bairros com ausência de notificação encontram-se em zona de alerta para a doença.

Os produtos gerados sobre o risco relativo e a distribuição espacial da doença possuem importância para auxiliar as políticas de gestão e controle da doença, sendo documentos para identificar as áreas vulneráveis e com necessidade de intervenção pelo poder público. Essa possibilidade é dada pelas técnicas de geoprocessamento para espacializar os resultados obtidos, permitindo melhor visualização das áreas agravadas.

\section{REFERÊNCIAS}

ARAUJO, R.V.; ALBERTINI, M.R.; COSTA-DA-SILVA, A.L.; SUESDEK, L.; FRANCESCHI, N.C.S.; BASTOS, N.M.; KATZ, G.; CARDOSO, V.A.; CASTRO, B.C.; CAPURRO, M.L.; ALLEGRO, V.L.A. C. São Paulo urban heat islands have a higher incidence of dengue than other urban áreas. Brazilian Journal of Infectious Diseases,Salvador, v. 19, n. 2, p. 146-155, 2015.

BARBOSA, G.L.; LOURENÇO, R.W. Análise da distribuição espaço-temporal de dengue e da infestação larvária no município de Tupã, Estado de São Paulo. Revista da Sociedade Brasileira de Medicina Tropical, Uberaba, v. 43, n.2, p.145-151, 2010.

BARBOSA, I. R.; SILVA, L. P. Influência dos determinantes sociais e ambientais na distribuição espacial da dengue no município de Natal-RN. Revista Ciência Plural, Natal, v. 1, n. 3, p. 62-75, 2015.

BAHTT, S.; GETHING, P.W.; BRADY, O.J.; MESSINA, J.P.; FARLOW, A.W.; MOYES, C.L.; DRAKE, J.M.; BROWNSTEIN, J.S.; HOEN, A.G.; SANKOH, O.; MYERS, M.F.; GEORGE, D.B.; JAENISCH, T.; WINT, G.R.; SIMMONS, C.P.; SCOTT, T.W.; FARRAR, J.J.; HAY, S.I. The global distribution and burden of dengue. Nature, Londres, v. 496, p. 504-507, 2013.

BRASIL. Ministério da Saúde. Secretaria de Vigilância em Saúde. Plano Nacional de Controle da Dengue. 2002. 32p. 
BRASIL. Ministério da Saúde. Secretaria de Vigilância em Saúde. Diagnóstico rápido nos municípios para vigilância entomológica do Aedes aegypti no Brasil - LIRAa: metodologia para avaliação dos índices de Breteau e Predial. 2005. 60p.

BRASIL. Ministério da Saúde. Secretaria de Vigilância em Saúde. Boletim Epidemiológico 2014. Vol. 45, n. 3, p. 1-4. 2014. Disponível em: <http://portalsaude.saude.gov.br/images/pdf/2014/maio/29/BE-201445--3--primeiras-semanas.pdf> Acesso em: 28 jul 2016.

BRASIL. Ministério da Saúde. Secretaria de Vigilância em Saúde. Departamento de Vigilância Epidemiológica. Sistema de Informação de Agravos de Notificação - SINAN, Brasília: Editora do Ministério da Saúde, 2015.

BRASIL. Ministério da Saúde. Secretaria de Vigilância em Saúde. Boletim Epidemiológico 2016. Vol. 47, n. 28, p. 1-10. Disponível em: <http://portalsaude.saude.gov.br/images/pdf/2016/julho/15/2016-boletimepi-n28-dengue-chik-zika-se23.pdf> Acesso em: 28 jul 2016.

ESRI. ECONOMIC AND SOCIAL RESEARCH INSTITUTE. ArcGIS 10.3. 2014.

FERNANDES, R.S.; NEVES, S.M.A.S.; NEVES, R.J. Risco da dengue nos municípios pantaneiros de Mato Grosso. Boletim Goiano de Geografia, Goiás, v. 34, n. 1, p. 93-110, 2014.

GONÇALVES, R.P. Contribuições recentes sobre conhecimentos, atitudes e práticas da população brasileira acerca da dengue. Revista Saúde e Sociedade, São Paulo, v. 24, n. 2, p. 578-593, 2015.

GUBLER, D.J. Dengue and dengue hemorrhagic fever: its history and resurgence as a global health problem. In: GUBLER, D. J., KUNO G. (Ed.) Dengue and dengue hemorrhagic fever. New York: CAB International, cap. 1, p. 1-22. 1997.

GUZMAN, M.G.; HALSTEAD, S.B.; ARTSOB, H.; BUCHY, P.; FARRAR, J.; GUBLER, D.J.; HUNSPERGER, E.; KROEGER, A.; MARGOLIS, H.S.; MARTÍNEZ, E.; NATHAN, M.B.; PELEGRINO, J.L.; SIMMONS, C.; YOKSAN, S.; PEELING, R.W. Dengue: a continuing global threat. Nature Reviews Microbiology, Londres, v. 8, n. 12, p. 7-16, 2010.

HASSAN, H.; SHOHAIMI, S.; HASHIM, N.R. Risk mapping of dengue in Selangor and Kuala Lumpur, Malaysia. Geospatial Health, Pavia, v. 7, n. 1, p. 21-25, 2012.

HONORATO, T.; LAPA, P.P.; VENDAS, C.M.; REIS-SANTOS, B.; TRISTÃO-SÁ, R.; BERTOLDE, A.I.; MACIEL, E.L. Spatial analysis of distribution of dengue cases in Espírito Santo, Brazil, in 2010: use of Bayesian model. Revista Brasileira de Epidemiologia,São Paulo, v. 17, p. 150-159.

HONORIO, N.A. Spatial evaluation and modeling of dengue seroprevalence and vector density in Rio de Janeiro, Brazil. Plos Neglected TropicalDiseases, San Francisco, v. 3, n. 11, p. e545, 2009.

IBGE - INSTITUTO BRASILEIRO DE GEOGRAFIA E ESTATÍSTICAS. Censo Demográfico 2010. Disponível em: <www.censo2010.ibge.gov.br>. Acesso em 14 jul 2016.

IBGE - INSTITUTO BRASILEIRO DE GEOGRAFIA E ESTATÍSTICAS. Cidades. Itú. Disponível em: <http://cod.ibge.gov.br/8BY> Acesso em: 25 jul 2016.

HORTA, M.A.P.; FERREIRA, A.P.; OLIVEIRA, R.B.; WERMELINGER, E.D.; KER, F.T.O.; FERREIRA, A.C.N.; CATITA, C.M.S. Os efeitos do crescimento urbano sobre a dengue. Revista Brasileira em Promoção da Saúde, Fortaleza, v. 26, n. 4, p. 539-547, 2014.

JOHANSEN, I.C.; CARMO, R.L.; ALVES, L.C. Desigualdade social intraurbana: implicações sobre a epidemia de dengue em Campinas, SP, em 2014. Cadernos Metrópole, São Paulo, v. 18, n. 36, p. 421$440,2016$.

LIMA, V.L.; ANDRADE, V.R..; RESTITUTTI, C.; CARMO, R.L.; SILVEIRA, N.Y.J. Controle integrado da dengue utilizando geoprocessamento. Superintendência de Controle de Endemias (SUCEN). Secretaria da Saúde de São Paulo. São Paulo, SP, Brasil, 2003.

LIMA, R.C.; MOREIRA, E.B.M.; NOBREGA, R.S. A influência climática sobre a epidemia dengue na cidade do Recife por Sistema de Informações Geográficas. Revista Brasileira de Geografia Física, Recife, v.9, n.2, p. 384-398, 2016.

MONDINI, A. Spatio-temporal tracking and phylodynamics of na urban dengue 3 outbreak in São Paulo, Brazil. Plos Neglected Tropical Diseases, Londres, v. 3, n. 5, p. e448, 2009. 
NASCIMENTO, M.C.; RODRIGUES JÚNIOR, A.L.; RODRIGUES, D.O. Configuração espacial da Dengue no contexto socioeconômico de Alfenas/MG: Retrato de uma década. Medicina, Ribeirão Preto, v. 48, n. 2, p. 181-189, 2015.

OLIVEIRA, A.F.M.; SOUSA, A.L.L.; SILVA, R.C.; CARMO FILHO, J.R. Estudo Ecológico da Dengue em Araguaína-TO entre 2001 e 2010. Estudos, Goiás, v. 42, n. 4, p. 517-526, 2015.

PEDROSO, L.B.; MOURA, G.G. Distribuição Espacial da Dengue no Município de Ituiutaba/MG, 20092010. Hygeia,Uberlândia, v. 8, n. 15, p. 119-136, 2012.

PEDROSO, L.B.; MENDES, P.C. A Influência de Aspectos Meteorológicos sobre as Notificações de Dengue em Uberaba/MG. Revista Geonorte, Manaus, v. 3, n. 9, p. 688-697, 2012.

PELISSARI, B. Aspectos socioambientais associados à ocorrência de dengue em um município do estado do Mato Grosso. Revista de Epidemiologia e Controle de Infecção, Santa Cruz do Sul, v. 6, n.1, 2016.

PEREIRA, M.; SUZUKI, A.; BISORDI, I.; BARLETA, C.; FERREIRA, I.T.R.N.; FROES, M.H.; MAEDA, A.Y.; CRUZ, M.G.B.; MARGARIDO, S.A.; SECCO, R.H.; GOMES, A.H.A.; WANDERLEY, D.M.V. Dengue no Estado de São Paulo: Situação epidemiológica e ações desenvolvidas em 2013. Boletim Epidemiológico Paulista, São Paulo, v. 10, n. 119, p. 3-14, 2013.

PREFEITURA MUNICIPAL DE ITU, 2015. Dados gerias da Estância Turística de Itu. Disponível em: < http://itu.sp.gov.br/site/?page_id=274.> Acesso em: 29 jan 2016.

REIS, C.B.; ANDRADE, S.M.O.; CUNHA, R.V. Responsabilização do outro: discursos de enfermeiros da Estratégia Saúde da Família sobre ocorrência de dengue. Revista Brasileira de Enfermagem, Brasilia, v. 66, n. 1, p. 74, 2013.

SÃO PAULO. SSE/CSAN. Secretaria de Estado de Saneamento e Energia. Apoio técnico à elaboração dos planos municipais de saneamento e elaboração do plano regional de saneamento para os municípios da Bacia Hidrográfica dos Rios Sorocaba e Médio Tietê - UGRHI 10. 2010. 115p.

SEADE - FUNDAÇÃO SISTEMA ESTADUAL DE ANÁLISE DE DADOS. Estado de São Paulo e suas regionalizações. 2015.

SILVA, D.C.C.; LOURENÇO, R.W.; CORDEIRO, R.C.; CORDEIRO, M.R.D. Análise da relação entre a distribuição espacial das morbidades por obesidade e hipertensão arterial para o estado de São Paulo, Brasil, de 2000 a 2010. Ciência \& Saúde Coletiva, Rio de Janeiro, v. 19, n. 6, p. 1709-1719, 2014.

SILVA, A.A.; MIRANDA, C.F.; FERREIRA, J.R; ARAÚJO, E.J.A. Fatores sociais e ambientais que podem ter contribuído para a proliferação da dengue em Umuarama, estado do Paraná. Acta Scientiarum Health Sciences, Maringá, v. 25, n. 1, p. 81-85, 2003.

SIMMONS, C.P.; FARRAR, J.J.; NGUYEN, V.V.; WILLS, B. Dengue. New England Journal of Medicine, v. 366, p. 1423-1432, 2012.

SOUZA, L.S.; BARATA, R.C.B. Diferenciais intraurbanos na distribuição de dengue em Cuiabá, 2007 e 2008. Revista Brasileira de Epidemiologia, São Paulo, v. 15, n. 4, p. 761-70, 2012.

TELLE, O. The spread of dengue in an endemic urban milieu-the case of Delhi, India. Plos One, Londres, p. $1-17,2015$.

TOLEDO, A.L.A.; ESCOSTEGUY, C.C.; MEDRONHO, R.A.; ANDRADE, F.C. Confiabilidade do diagnóstico final de dengue na epidemia 2001-2002 no Município do Rio de Janeiro, Brasil. Cadernos de Saúde Pública, São Paulo, v. 22, n. 5, p. 933-940, 2006.

WHEN, T.H. Incorporating the human-Aedes mosquito interactions into measuring the spatial risk of urban dengue fever. Applied Geography, Estados Unidos, v. 62, p. 256-266, 2015. 